\title{
Development Status of an Intense Beam Klystron
}

\author{
P. D. Coleman ${ }^{*}$, K. J. Hendricks ${ }^{\dagger}$, M. J. Arman ${ }^{\dagger}$, \\ L. A. Bowers ${ }^{\dagger}$, and R. W. Lemke* \\ * Sandia National Laboratories, Albuquerque, NM 87185 \\ ${ }^{\dagger}$ Phillips Laboratory, Kirtland AFB, Albuquerque, NM 87117
}

\begin{abstract}
Investigations are being performed on a high current $(16 \mathrm{kA})$, mildly relativistic
. ( $(400 \mathrm{kV})$, L-band klystron source: Experiments are in an early stage; and thus far have progressed to beam modulation studies. This paper discusses general klysiron design considerations, beam propagation results, initial modulation results, and various extraction techniques being considered.
\end{abstract}

\section{INTRODUCTION}

The Phillips Laboratory and Sandia National Laboratories are currently investigating operation of an L-band klystron which uses an annular intense relativistic electron beam (IREB). The intense space charge in the beam can be used to decrease the drift distance needed to reach maximum bunching, enhance the bunching process at large modulation levels, and provide electrostatic insulation of the rf gaps $(1,2)$. Work on this type of klystron began at the Naval Research Laboratory (NRL) and is currently in progress at NRL, Phillips Laboratory (PL), Los Alamos National Laboratory (LANL), and Physics International (PI) $(3,4,5,6)$.

Typically this type of klystron has been operated with beam impedances $\left(V_{\text {diode }}\left(I_{\text {beam }}\right)\right.$ of $30-100 \Omega$ The effort described here is intended to further investigate low beam impedance ( $25 \Omega$ in this case) operation of the klystron. Operation with very low beam impedances allows generation of very high beam powers $(>5$ GW) with modest diode voltages.

\section{Klystron Parameters}

The klystron presently under development by Phillips and Sandia is known as the Annular Beam Amplifier (ABA).The table below lists design specifications and current operation points for this klystron.

\section{DISCLAIMER}

This report was prepared as an account of work sponsored by an agency of the United States Government. Neither the United States Government nor any agency thereof, nor any of their employees, makes any warranty, express or implied, or assumes any legal liability or responsibility for the accuracy, completeness, or usefulness of any information, apparatus, product, or process disclosed, or represents that its use would not infringe privately owned rights. Reference herein to any specific commercial product, process, or service by trade name, trademark, manufacturer, or otherwise does not necessarily constitute or imply its endorsement, recommendation, or favoring by the United States Government or any agency thereof. The views and opinions of authors expressed herein do not necessarily state or reflect those of the United States Government or any agency thereof. 


\section{DISCLAIMER}

Portions of this document may be illegible in electronic image products. Images are produced from the best available original document. 
TABLE 1. ABA Klystron Specifications

\begin{tabular}{lll} 
Parameter & $\begin{array}{l}\text { Design } \\
\text { Specification }\end{array}$ & $\begin{array}{l}\text { Current } \\
\text { Operation }\end{array}$ \\
\hline frequency $(\mathrm{GHz})$ & 1.3 & 1.3 \\
$\mathrm{~V}_{\text {diode }}(\mathrm{kV})$ & 400 & 400 \\
$\mathrm{I}_{\text {beam }}(\mathrm{KA})$ & 16 & $6-16$ \\
modulation $=I_{\mathrm{If}} / I_{\mathrm{dc}}(\%)$ & 70 & $15-30$ \\
$P_{\text {out }}(\mathrm{GW})$ & 2.0 & not attempted yet \\
pulse width $(\mu \mathrm{s})$ & 1.0 & 0.3 \\
.rep rate & single shot & single shot \\
\hline
\end{tabular}

\section{Hardware Description}

Figure 1 shows a schematic of the tube. The diode is driven by a $5 \Omega, 300 \mathrm{~ns}$, $400 \mathrm{kV}$ pulse forming line. The cathode consists of a $14.4 \mathrm{~cm}$ diameter bare stainless steel surface that explosively field emits (7) an annular beam. The shape of the cathode was chosen to eliminate backscattered electrons. The beam is guided into a $15.4 \mathrm{~cm}$ diameter drift tube using an $8 \mathrm{kG}$ solenoidal field.

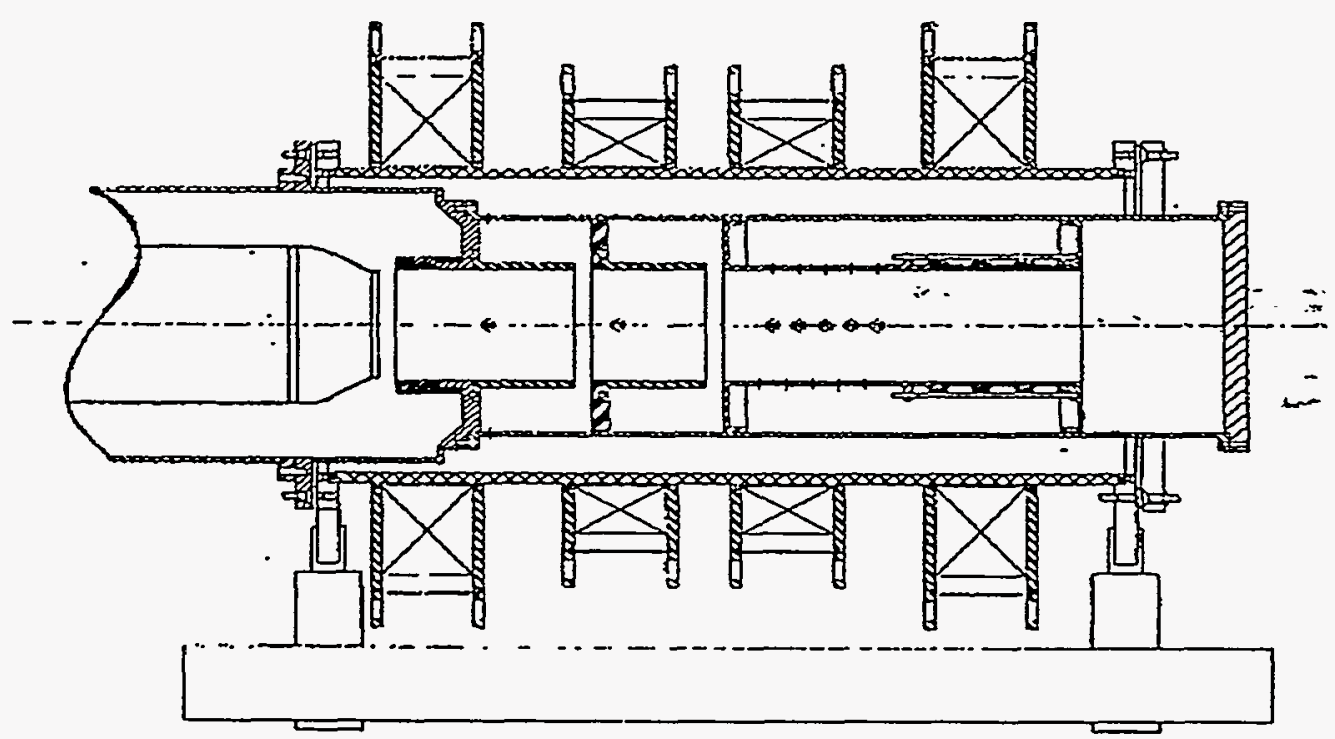

FIGURE 1. Schematic of the klystron tube.

At present there are two of cavities, a modulating cavity and one idler cavity. (In the near future extraction hardware, and possibly an additional idler cavity, will be 
added.) Both cavities are $3 \lambda_{i} 4$ coaxial resonators (coaxial impedance of $31 \Omega$ ). The modulator cavity is driven with a capacitively coupled coaxial feed. A 500 $\mathrm{kW}, 2 \mu \mathrm{s}$ magnetron oscillator is used as the driving source.

Downstream of the idler cavity is an axial and azimuthal array of B-dot probes. The probes have been calibrated at both video and if frequencies. With the appropriate filtering, these probes can be used to monitor $\mathrm{dc}$ and if currents as a function of drift distance.

The following section will discuss analytical and particle in cell (PIC) simulations of the tube, which give an indication of the expected performance. Next, experimental results are discussed. These include beam propagation, beam modulation, gap loading effects, and feedback studies. Various extraction techniques that are being considered will then be reviewed. Finally an overall summary of the : status of the program is presented... .

\section{THEORY AND SIMULATIONS}

\section{Beam Issues}

The space charge in the intense beam is a very important part of the operation of this tube. This can be seen by reviewing the energy distribution for a dc beam (2). When the beam is injected into the drift tube, its total energy is divided between kinetic and potential energies. As the beam current increases, its potential energy increases and its velocity decreases. Because of this effect, a space charge limiting current exists (8).

An annular beam is used in this experiment. This helps minimize the beam's potential energy by moving the beam's space charge close to the grounded wall. As an example of the magnitude of some of these quantities, consider the beam parameters for this klystron: $\mathrm{r}_{\mathrm{w}}=7.7 \mathrm{~cm}, \mathrm{r}_{\mathrm{b}}=6.9 \mathrm{~cm}, \mathrm{I}_{\text {beam }}=16 \mathrm{kA}$, and $\mathrm{V}_{\text {diode }}=$ $400 \mathrm{kV}$. The limiting current is calculated to be $\mathrm{I}_{\mathrm{scl}}=25 \mathrm{kA}$. The total, kinetic, ând potential energies are found to be $400 \mathrm{keV}, 258 \mathrm{keV}$, and $142 \mathrm{keV}$ respectively. Hence, even with the annular geometry, the potential energy of the beam represents a very significant fraction of the total beam energy.

\section{Small Signal Space Charge Waves}

The dispersion relation for space charge waves on a drifting beam can be expressed as (9)

$$
(\omega-k v)^{2}=\omega_{q}^{2} / \gamma^{2}
$$


where $\mathrm{v}=\beta c=$ drift velocity of the beam, $\gamma=\left(1-\beta^{2}\right)^{-1 / 2}, k=2 \pi / \lambda$, $\omega_{q}^{2}=R^{2} \omega_{p}^{2}$, and $\omega_{p}^{2}=\frac{n e^{2}}{\varepsilon_{o} \gamma m}=$ square of the plasma frequency. $\mathrm{R}$ is known as the plasma reduction factor $(10)$ and is purely a geometrically determined quantity (for the case being discussed here, $\mathrm{R}=0.15$ ).

The rf current downstream of the modulating gap can be expressed as (2)

$$
I_{1}(z, t)=\frac{V_{g a p}}{Z} \sin (\Delta k z) e^{i(\omega t-k z-\pi / 2)}
$$

where $V_{\text {gap }}=$ modulating gap voltage, $Z$ = effective beam impedance which is a function of the $d \mathrm{dc}$ beam parameters discussed above, $: \Delta k=\omega_{q} / \gamma v$, and $k=\omega / v$. Equation 2 suggests that the modulation will peak at $z=(\pi / 2) / \Delta k$ (one quarter plasma wavelength) and that the wave's phase velocity is $\omega / k=v$.

\section{Simulations}

As the beam modulation increases, the physics of the bunching process becomes nonlinear. Computer simulations are used to predict the physics in this regime.

MAGIC, a 2.5 D relativistic electromagnetic particle in cell code, solves for the particle orbits and electromagnetic fields self consistently. Figure 2 displays the results of MAGIC simulations for the case of an imposed gap voltage of $40 \mathrm{kV}$ on the modulating gap. The modulating gap is located at $\mathrm{z}=0 \mathrm{~cm}$ and the idler gap at $z=15 \mathrm{~cm}$.

Also displayed in the figure are calculations by the code BUNCHER (11). This code uses a single particle phase model that includes relativistic, space charge, large signal and transit time effects. Both computer predictions show similar beam modulation growth. The modulation peaks at $70 \%$, approximātely $20 \mathrm{~cm}$ downstream of the idler gap. Ideally, the extraction hardware would be placed at this location. 


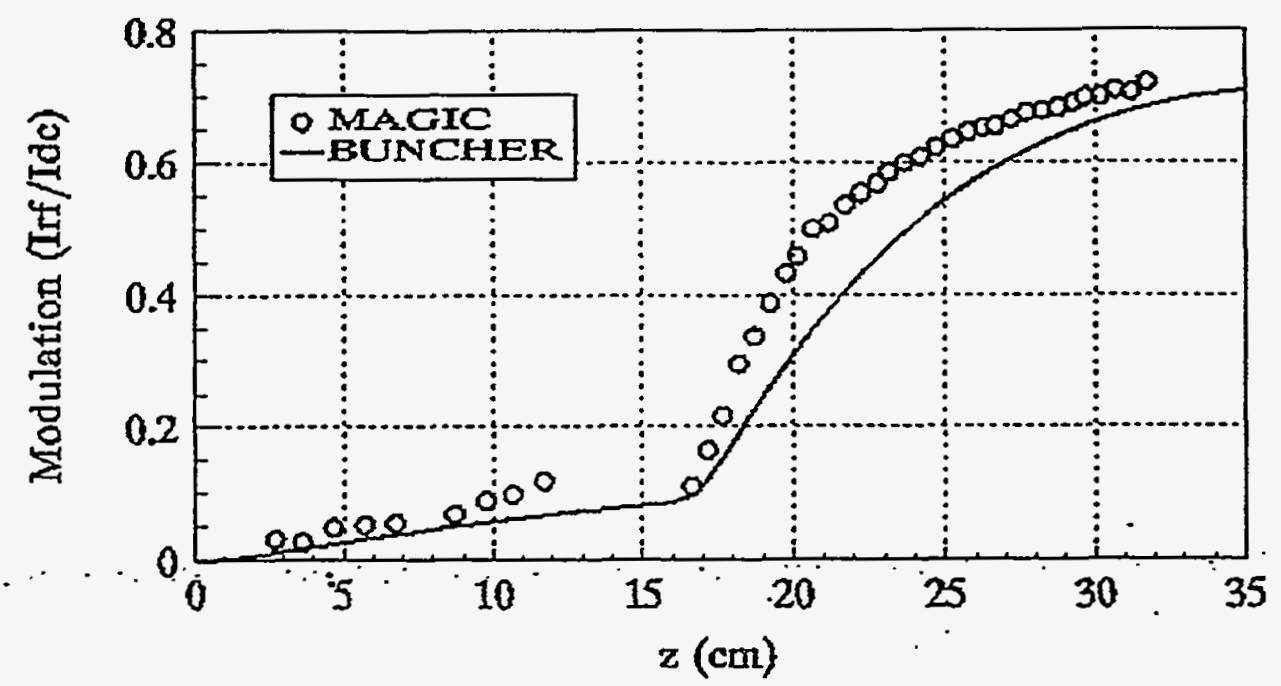

FIGURE 2. Code predictions of modulation coefficient as a function of drift distance. First gap at $0 \mathrm{~cm}$, second gap at $15 \mathrm{~cm}$. Pararneters: $V_{\text {diode }}=400 \mathrm{kV}, l_{\text {beam }}=16 \mathrm{kA}, V_{\text {gap } 1}=$ $40 \mathrm{kV}, \mathrm{V}_{\mathrm{gap} 2}=280 \mathrm{kV}$.

\section{EXPERIMENTAL RESULTS}

\section{Beam Propagation}

Initial experiments concentrated solely on dc beam propagation through the smooth walled system (i.e. no If cavities). The A-K gap was adjusted to yield a 16 $\mathrm{kA}$ current for the imposed $400 \mathrm{kV}$ diode voltage. The cathode.was positionéd at a slightly converging point in the $8 \mathrm{kG}$ applied solenoidal field. This resulted in the beam being compressed from the cathode radius of $7.2 \mathrm{~cm}$ to approximately 6.9 $\mathrm{cm}$.

Rogowski coils and B-dot probes were used to verify that no beam loss occurred for the beam propagating the full length of the structure $(70 \mathrm{~cm})$. Burn marks on the beam stop indicated that the beam thickness was $5 \mathrm{~mm}$ and that the outer beam radius was $5 \mathrm{~mm}$ from the drift tube wall.

\section{Modulation Growth}

The initial If experiments began by characterizing the beam modulation. The modulation cavity was excited with an external magnetron source. The B-dot array was then used to map the if current growth as a function of distance downstream of the rf gaps (see Figure 3). The modulation grows as the beam propagates from the 
modulating cavity $(z=0 \mathrm{~cm})$ to the idler cavity $(z=15 \mathrm{~cm})$. At this point the if current excites the idler gap resulting in increased modulation growth.

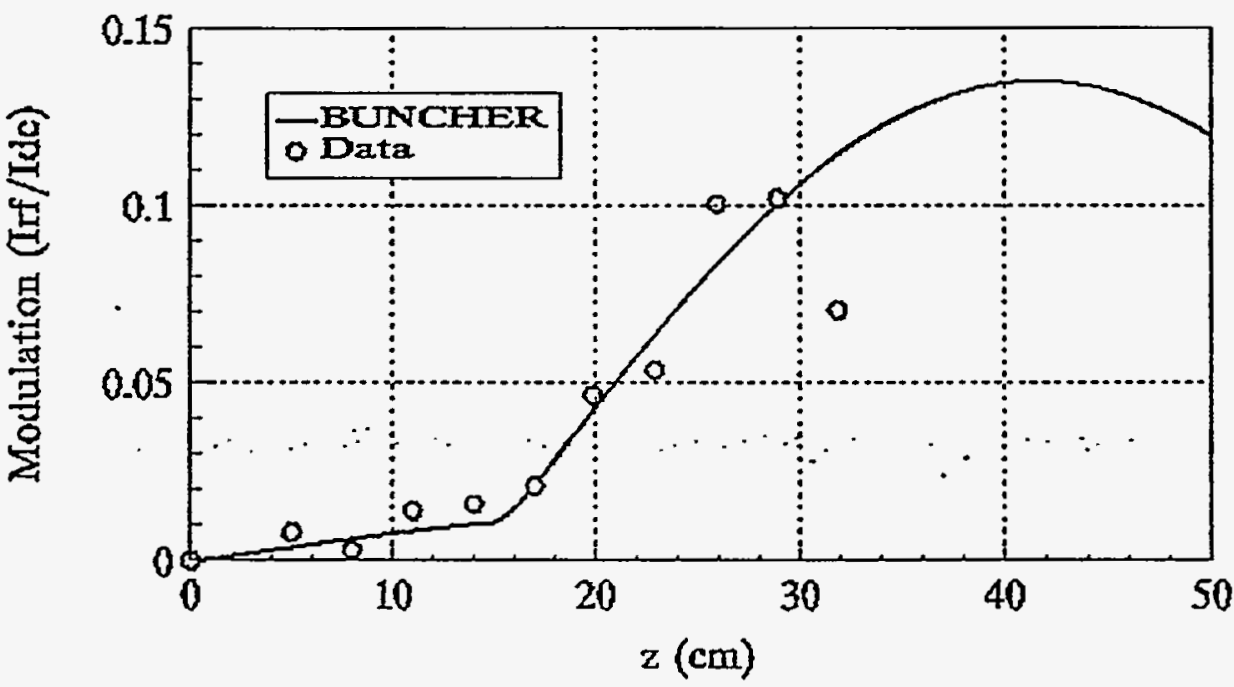

FIGURE 3. Measured beam modulation as a furction of drift distance. Measured parameters: $V_{\text {diode }}=400 \mathrm{kV}, \mathrm{I}_{\text {beam }}=12 \mathrm{kA}, V_{\text {gap } 1}=7 \mathrm{kV}, V_{\text {gap2 }}=15 \mathrm{kV}$. BUNCHER code predictions are shown as solid line.

The small signal theory above suggests that the modulation should peak at 23 $\mathrm{cm}$ downstream of the modulating cavity. Also shown in the figure are BUNCHER code predictions assuming a gap voltage of $7 \mathrm{kV}$ on the modulator gap, and $40 \mathrm{kV}$ on the idler gap. With these assumed voltages, the code agrees fairly well with the data. However, calibrated E-dot probes in the idler cavity indicate that only. $15 \mathrm{kV}$ was present on the gap.

The peak modulation for this set of data only reached $10 \%$ compared to the goal of $70 \%$. Supplying some feedback was investigated as a way to increase the modulation (see below). In addition, it is planned to insert a second idler cavity which should further boost the modulation.

\section{Gap Loading}

Calibrated E-dot probes were mounted in both the modulation and idler cavities. Assuming a given mode structure (found from SUPERFISH calculations), one can infer a gap voltage from these probes. Figure 4 shows the gap voltage waveforms for the case of a $12 \mathrm{kA}$ injected beam. The.modulating gap voltage rises as the magnetron fills the cavity. When the beam arrives the voltage quickly decays away. The idler cavity voltage, on the other hand, rises as the modulated beam excites it. 

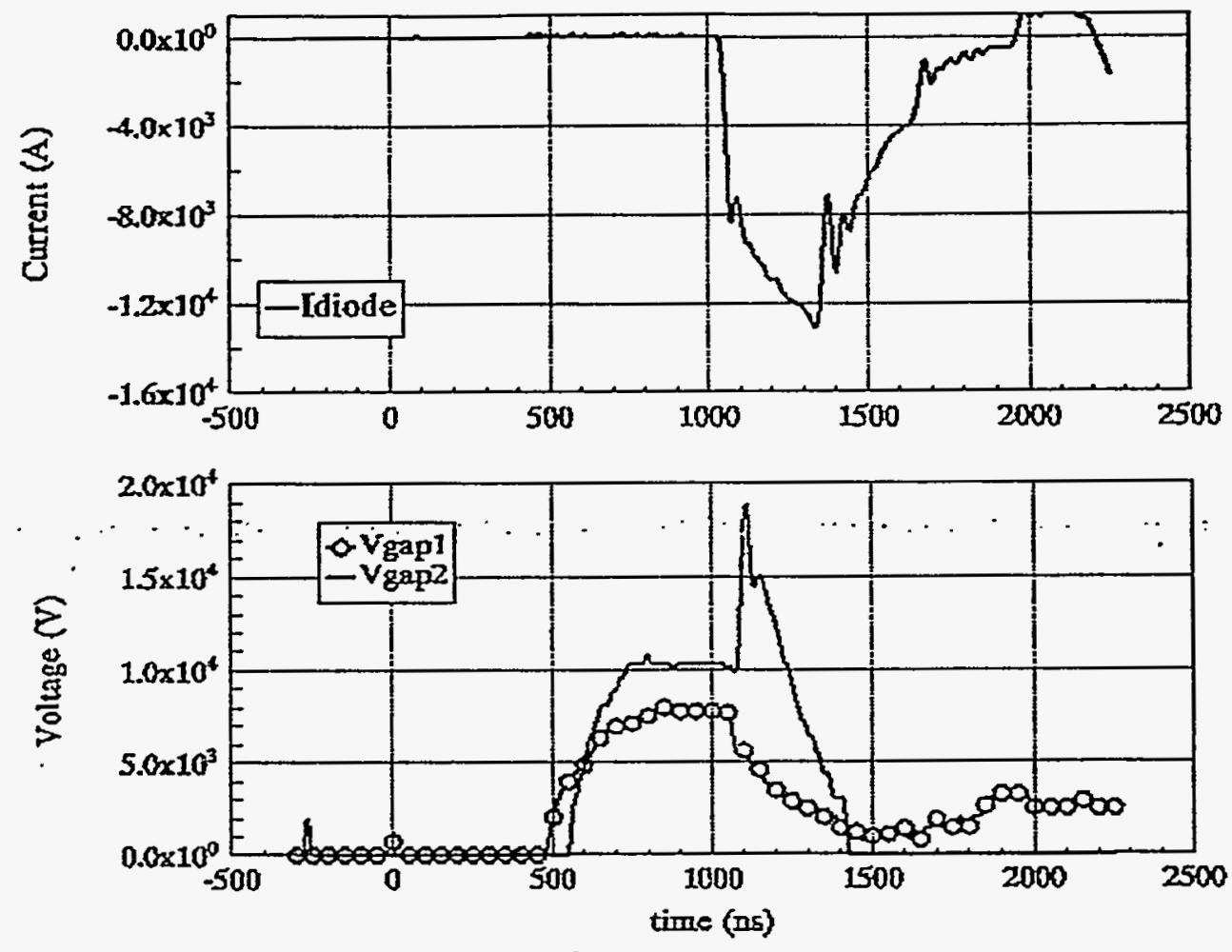

FIGURE 4. Modulator and idler gap voltages showing beam loading effects.

The decay in the modulating voltage appears to be related to the very high current extracting energy out of the cavity faster that it can be replenished by the driving magnetron. A comparison of the dc beam impedance $\left(V_{\text {diode }} / I_{d c}\right)$ and the if gap impedance $\left(V_{g a p}^{2} / 2 P\right.$, where $\mathrm{P}$ is the net rf power into the cavity derived from a directional coupler) has been made. The agreement between the two waveforms suggests the gap voltage is dominated by the loading by the beam.

\section{Feedback Studies}

The initial amplifier experiments showed a low peak modulation and a drop off of the modulation due to beam loading. In an attempt to increase the tube's gain and possibly improve these problems, feedback was introduced between the cavities. The simplest technique for providing the feedback was to cut irises in the common wall between the cavities.

The magnitude of the feedback is determined by the size of the coupling slots. The phase of the feedback relative to the space charge wave is also a critical parameter. Measurements were therefore made of the wave's phase advance down- 
stream of the modulating cavity. The phase velocity of the wave was measured to be $v / c=0.8 \mathrm{c}$ (quite close to the beam velocity of $0.81 \mathrm{c}$ as predicted by the small signal space charge wave theory above). The phase advance at the location of the idler gap was approximately $380^{\circ}$, which was close to the ideal delay of $360^{\circ}$.

Experiments incorporating this feedback were performed with a $400 \mathrm{kV}, 7 \mathrm{kA}$ injected beam. Figure 5 shows the gap voltages recorded for this case. The modulating voltage is seen to have a different qualitative behavior from the uncoupled case (see Figure 4). The voltage now grows after the arrival of the beam, and the general nature of the tube is similar to that of a driven oscillator. Measurements of the rf current downstream of the idler cavity showed a peak modulation of 20 to $30 \%$ was achieved.
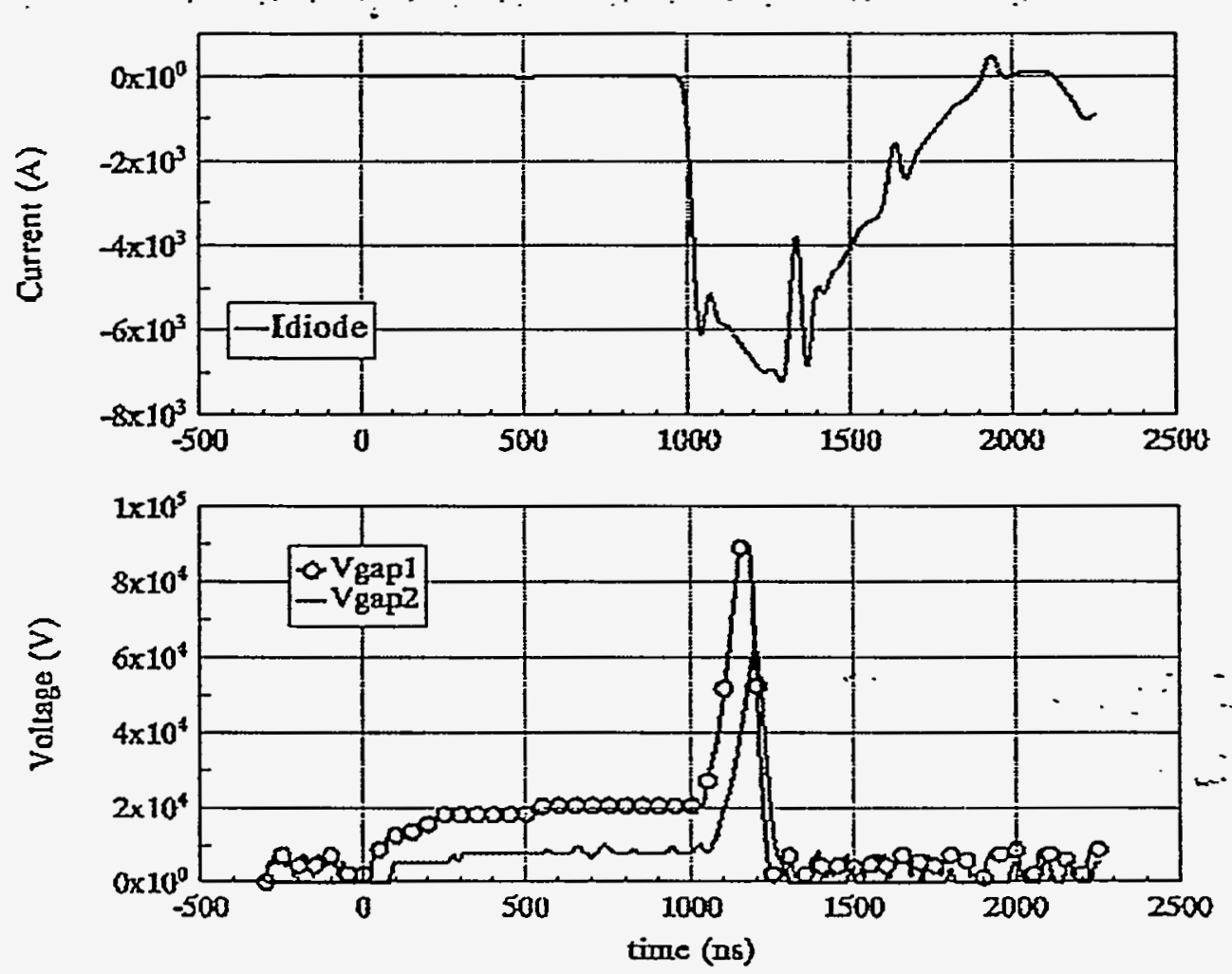

FIGURE 5. Modulator and idler gap voltages for coupled cavity operation.

The rf current grew for the first 150 ns of the beam pulse and then abruptly shut down. Coincident with the pulse termination, a $2 \mathrm{GHz}$ oscillation and an $\mathrm{x}$-ray spike were observed. The presence of the high frequency signal suggest the onset of a parasitic oscillation. If this is indeed found to be true and a higher order mode can be identified, mode damping techniques may be used to suppress the oscillation. 


\section{EXTRACTOR DISCUSSIONS}

Thus far in the program, experiments have concentrated on beam modulation studies. The next stage of experiments will focus on extracting power from the modulated beam. Various extractor designs and issues are now being considered. The general design of the extractor can be guided by the desire for a low shunt impedance $\left(R_{S}\right)$, high $Q$ output structure. A low $R_{S}(<16 \Omega$ for this experiment) avoids the high current beam inducing too high a gap voltage. If the voltage exceeds a threshold level, electrons would be reflected back upstream, thereby disrupting the operation of the tube. A narrowband structure more efficiently extracts the power at the desired fundamental frequency.

The advantages of a low shunt impedance and high $Q$ imply one should design a structure with a low characteristic impedance $\rho=R_{s} / Q$. The characteristic impedance of the structure is set solely by its geometry, and can be expressed as

$$
\rho=R_{s} / Q=\frac{V_{g}^{2}}{2 \omega U}=\frac{T^{2}\left[\int E d z\right]^{2}}{2 \omega U}
$$

where $\mathrm{U}=$ stored energy, and $T=\left|\int E e^{i k z} d z\right| / \int E d z$ is the transit time factor. (It is important to note that for the large diameter drift tube considered here, $T=0.5$ and hence has a significant effect on $\rho$.)

The extractor design philosophy is then to first choose a structure with a low $\rho$. The efficiency of the extractor can then be optimized by operating with a high $Q$ (within the constraint of not exceeding the reflexing voltage threshold).

The optimum extractor design is still under investigation, and several approaches are being considered. Single gap extractor designs are being used on intense beam klystrons at NRL and LANL $(12,13)$. Both structures extract power onto a coaxial transmission line. LANL uses a pill box cavity with a very low $Q$ of approximately 4 . NRL's design resembles that of a very heavily loaded coaxial cavity.

The disadvantage of the single gap extractor is that the entire retarding potential must be developed across a single gap. This leads to high field stress and the potential for rf breakdown at high powers. Another type of extractor being studied uses a distributed interaction space. Here the retarding potential is spread over a longer distance, thereby reducing field stress. An example of this type structure would be the traveling wave output circuit used on the LLNL RKA (14). NRL has also been experimenting with what is described as an inductively loaded wide gap (15). Here inductive rods are shunted across a wide $(\equiv \lambda / 2)$ gap in order to avoid dc potential well problems for the beam. 
An additional concern that arises when considering the extractor design is that of the potential and kinetic energy distribution in the beam. The intense modulated beam will have much of its total energy in potential energy. Since one can only extract If power from the kinetic energy of the beam, this can impose an overall efficiency limit on the extractor. A possible technique for converting some of the beam's potential energy back into kinetic is to gradually introduce a dc grounded center conductor into the extractor geometry. An example of this triaxial type geometry is shown in Figure 6.

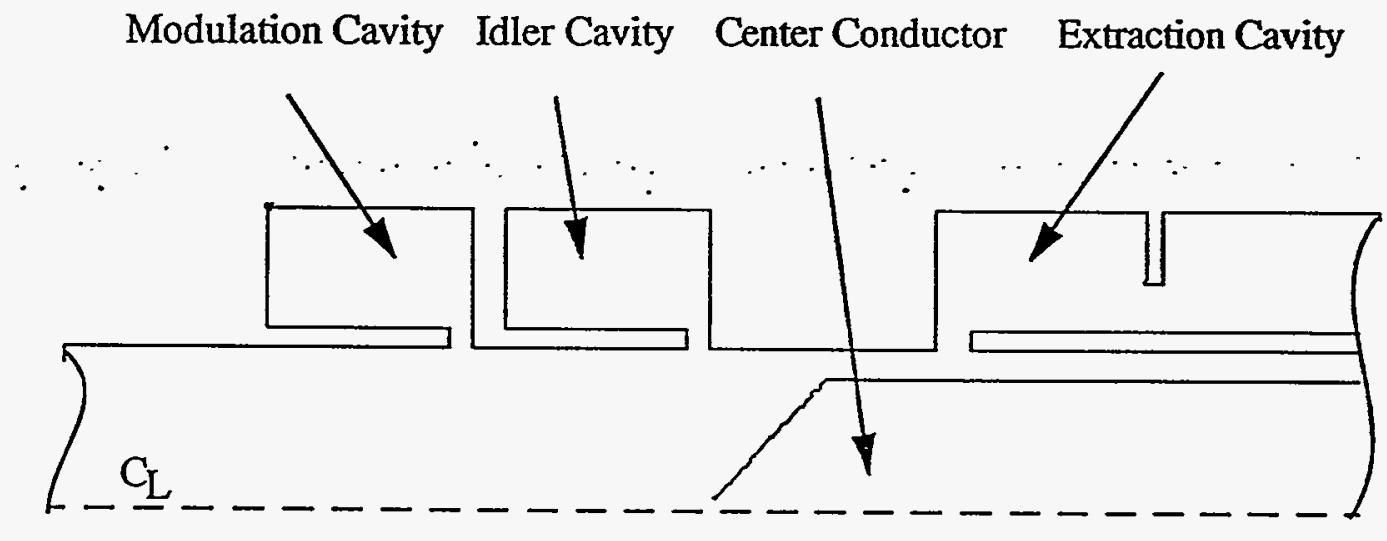

FIGURE 6. Example of a triaxial output extractor.

By bringing the additional grounded wall near the beam its potential energy is reduced and, by conservation of energy, its kinetic energy increased. PIC simulations are being used to estimate the distribution of particle energies for the modulated beam with and without the center conductor present. This information will then be used in conjunction with if codes such as SUPERFISH and CFISH (10) to design the extractor hardware.

\section{SUMMARY}

Work is under way to build and test a very high current, relativistic klystron. Initial experimental results have characterized beam propagation and modulation performance. Problems include drop off of the beam modulation due to the heavy beam loading and insufficient modulation levels. Future work will include adding a second idler cavity to be used in further modulating the beam. Extraction experiments are expected to begin in December of 1994.

\section{ACKNOWLEDGMENTS}

The authors wish to thank W. B. Haynes of LANL for his insighits into extractor designs. Also, the excellent work provided by the Phillips Lab and Sandia Lab technicians in support of the experimental effort is greatly appreciated. This work 
supported by the United States Department of Energy under contract DE-ACO494AL85000.

\section{REFERENCES}

1. M. Friedman and V. Serlin, "Modulation of intense relativistic electron beams by an external microwave source," Phys. Rev. Lett., vol. 55, pp. 2860-2863, 1985.

2. Y. Y. Lau, M. Friedman, J. Krall, and V. Serlin, "Relativistic klystron amplifiers driven by modulated intense relativistic electron beam," IEEE Trans. Plasma Sci., vol. 18, no. 3, June 1990.

3. V. Serlin and M. Friedman, "External modulation of intense relativistic electron beams with spatial and velocity inhomogeneities," Appl. Phys. Lett, vol. 62, no. 22, p. 2772, May 1993.

4. K. Hendricks, et. al., "Modulation of an annular electron beam by a two cavity klystron amplifier," presented at the IEEE Int. Conf. on Plasma Sci., Santa Fe, NM, June 1994.

5. M: V. Fazio, W. B. Haynes, B. E. Carlsten, R. M. Stringfield, "One microsecond pulse-width annular beam relativistic klystron operating at $500 \mathrm{MW}$," presented at the IEEE Int. Conf. on Plasma Sci., Santa Fe, NM, June 1994.

6. J. S. Levine and B. D. Harteneck, "Long pulse and high repetition rate operation of a relativistic klystron amplifier," presented at the IEEE Int. Conf. on Plasma Sci., Santa Fe, NM, June 1994.

7. R. B. Miller, K. R. Prestwich, J. W. Poukey, and S. L. Shope, "Production of annular electron beams by foilless diodes," J. Appl. Phys., vol 51, no. 7, p. 3506, July 1980.

8. R. B. Miller, Intense Charged Particle Beams, New York: Plenum Press, 1982, p. 84.

9. J. D. Lawson, The Physics of Charged-Particle Beams, Oxford, Claredon Press, 1988, p. 311.

10. G. M. Branch and T. G. Mihran, "Plasma frequency reduction factors in electron beams," IRE Trans. Elec. Dev., pp. 3-11, 1955.

11. R. W. Lemke, M. C. Clark, and B. M. Marder, "Theoretical and experimental investigation of a method for increasing the output power of a microwave tube based on the split-cavity oscillator," J. Appl. Phys., vol 75, no. 10, May 1994.

12. M. Friedman, V. Serlin, Y. Y. Lau, and J. Krall, "Relativistic klystron amplifier I - high power operation," in Proceedings of the SPIE Intense Microwave and Particle Beams II, vol. 1407. p. 2. 1991.

13. D. G. Rickel, et. al., "Development of a long-pulse $1.3 \mathrm{GHz}$ relativistic klystron amplifier," IEEE Trans. Plasma Sci., vol. 20, no. 3, p.373, June 1992.

14. R. D. Ryne, S. S. Yu, "Using traveling wave structures to extract power from relativistic klystrons," in Proceedings of the 1990 Linear Accelerator Conference, Sept. 1990.

15. M. Lampe, R. Hubbard, M. Friedman, and V. Serlin, "Inductively-loaded extended gaps for relativistic klystrons," presented at the IEEE Int. Conf. on Plasma Sci., Santa Fe, NM, June 1994.

16. J. H. Billen and L. M. Young, "POISSON/SUPERFISH on PC compatibles," in Proceedings of the 1993 Particle Accelerator Conference, May 1993. 